\title{
Análise vocal na infância: uma revisão integrativa
}

\author{
Vocal analysis in children: \\ an integrative review
}

\section{Análisis Vocal en la infancia: una revisión integradora}

\author{
Lorena de Almeida Ramos* \\ Bárbara Oliveira Souza* \\ Ana Cristina Côrtes Gama*
}

\section{Resumo}

Objetivo: realizar uma revisão integrativa de literatura sobre as características de vozes normais e disfônicas e a prevalência de lesões nas pregas vocais em crianças. Métodos: trata-se de uma revisão integrativa da literatura. Foi realizado o levantamento dos artigos cientificos por meio das bases de dados Medline, LILACS, IBECS, PubMed e ISI Web of Science. Na busca dos artigos foram utilizados os descritores "Distúrbios da Voz", "Disfonia", "Rouquidão", "Qualidade da Voz", "Acústica da Fala", "Voz", "Fonação", "Pregas Vocais", "Pediatria", "Criança", "Pré-Escolar". Para a análise dos dados, as informações coletadas foram organizadas de maneira concisa em um banco de dados, realizado de forma descritiva, considerando as informações referentes à amostra, objetivos, metodologia e resultados principais, e organizadas por similaridade de conteúdo. Resultados: foram identificados 770 artigos, dos quais 36 estavam diretamente relacionados ao objetivo da revisão e foram analisados. Conclusĩes: a qualidade vocal das crianças sem alterações vocais é caracterizada por valores de frequência fundamental que decrescem com o aumento da idade e com diferenças em relação ao sexo. O tempo máximo de fonação aumenta com a idade. Em crianças disfônicas a qualidade vocal é soprosa e rugosa, com grau de alteração de leve a moderado; as medidas acústicas da voz, os valores de tempo máximo de fonação estão alterados; frequência fundamental é reduzida ou os valores de frequência fundamental observados em crianças com alteração foram mais baixos do que os valores da normalidade. A lesão de prega vocal mais prevalente até seis anos de idade é o cisto e, após esta idade, é o nódulo vocal.

Palavras-chave: Distúrbios da Voz; Disfonia; Qualidade da Voz; Voz; Criança.

* Universidade Federal de Minas Gerais - UFMG - Belo Horizonte (MG), Brasil.

Contribuição dos autores: LAR foi responsável pela busca bibliográfica, desenvolvimento do texto, elaboração do projeto e do manuscrito; BOS foi responsável pela busca bibliográfica e desenvolvimento do texto; ACCG foi responsável pela orientação geral das etapas de execução e elaboração do projeto e do manuscrito.

Email para correspondência: bbarbara.oliveira@gmail.com Recebido: 30/04/2016

Aprovado: 13/02/2017 


\section{Abstract}

Objective: to perform an integrative literature review about the characteristics of normal and dysphonic voices and the prevalence of vocal cords lesions in children. Methods: it is a literature integrative review. The survey of scientific articles was carried out through data bases Medline, LILACS, IBECS (through the VHL Search Portal), PubMed and ISI Web of Science. In the search of the articles were used the descriptors "Voice Disorders", "Dysphonia", "Hoarseness", "Voice Quality", "Speech Acoustics", "Voice", "Phonation", "Vocal Cords", "Pediatrics", "Child", "Child Preschool". For the analysis of the data, the information collected was organized in a concise manner in a database, being performed in a descriptive way, considering the information about the sample, objectives, methodology and main results, and organized by content similarity. Results: 770 articles were identified, of which 36 were directly related to objective of the review and were analyzed. Conclusions: the vocal quality of children without vocal changes is characterized by fundamental frequency values that decrease with increase of age and with differences in relation to gender. The maximum phonation time increases with age. In dysphonic children the vocal quality is blow and rough, with a grade of alteration from mild to moderate; the acoustic measures of the voice, the maximum phonation time values are altered; the fundamental frequency is severe. The cyst is vocal cords lesion most prevalent up to six years age, and after this age is the vocal nodule.

Keywords: Voice Disorders; Dysphonia; Voice Quality; Voice; Child.

\section{Resumen}

Objetivo: Realizar una revisión integradora de la literatura sobre las características de vozes normales y disfonicas y la prevalencia de lesiones de las cuerdas vocales en niños. Métodos: se realizó una investigación integrativa de la literatura a través de articulos cientificos enontrados en las bases de datos Medline, LILACS IBECS, PubMed y ISI Web of Science. En busca de artículos se utilizaron los descriptores "Trastornos de la Voz", "Disfonía", "Ronquera", "Calidad de la Voz", "Acústica del Habla", "Voz", "Fonación", "Pliegues Vocales", "Pediatría”, "Niño", "Preescolar". Para el análisis de datos, la información recogida se organizó de manera concisa en una base de datos realizada de una manera descriptiva, teniendo en cuenta la información sobre la muestra, objetivos, metodología y resultados principales, organizados por la similitud del contenido. Resultados: fueron identificados 770 artículos de los cuales 36 estaban directamente relacionadas con el objetivo de la revisión y fueron analizados. Conclusiones: La calidad vocal de los niños sin trastornos vocales se caracteriza por la disminución de la frecuencia vocal fundamental con el aumento de la edad y con diferencias en relación al sexo. El tiempo máximo de fonación aumenta con la edad. Los niños con disfonía tienen una calidad de voz susurrante y rugosa, con un grado de alteración de leve a moderada; las medidas acústicas de la voz, y los valores de tiempo máximo de fonación están alterados. La frecuencia fundamental es reduzida o los valores de frecuencia fundamental obsevados en niños com alteración, fueron mas bajos que los valores de normalidad. La lesión de pliega vocal más prevalente hasta seis años de edad es el quiste. Después de esta edad son los nódulos vocales.

Palabras clave: Trastornos de la Voz; Disfonía; Calidad de la Voz; Voz; Niño.

\section{Introdução}

A prevalência de alterações de voz em crianças é de $6 \%$ a $38 \%$ sendo que, dentre essas, $92 \%$ podem ter o comportamento vocal inadequado como fator etiológico das disfonias, uma vez que as lesões de pregas vocais mais incidentes na população pediátrica são de base organofuncional ${ }^{2}$. O nódulo (53\%) e o cisto (21\%) estão presentes em $67 \%$ das crianças com queixas vocais. As fendas glóticas do tipo triangular posterior $(11 \%)$, médio-posterior $(39 \%)$ e dupla (17\%) também possuem alta prevalência na população infantil ${ }^{1}$.

As laringes de crianças e mulheres jovens apresentam valores menores de proporção glótica quando comparadas às dos adultos do sexo mascu- 
lino. Entretanto, a proporção glótica infantil ainda apresenta valores inferiores aos encontrados no sexo feminino, o que a torna propensa à abertura posterior em fenda triangular, onde se concentra a maior parte da energia vibratória durante a fonação. Em virtude disso, sugere-se uma alta suscetibilidade da população pediátrica para desenvolver lesões por fonotrauma no terço médio das pregas vocais ${ }^{3,4}$.

Apesar das particularidades da voz infantil e da alta prevalência de disfonia na infância, os estudos que descrevem as características vocais e laríngeas das crianças são escassos e necessitam de análise para uma melhor compreensão e abordagem da clínica vocal com a população pediátrica.

O objetivo do presente trabalho foi realizar uma revisão integrativa da literatura sobre as características de vozes normais e disfônicas e a prevalência de lesões nas pregas vocais em crianças.

\section{Método}

Trata-se de uma revisão integrativa da literatura sobre a qualidade vocal na infância. A metodologia empregada seguiu as seis fases propostas na literatura para a elaboração da revisão integrativa, a saber: 1) elaboração da hipótese, 2) busca na literatura, 3) definição das características dos estudos, 4) análise crítica dos estudos incluídos, 5) análise dos resultados e 6) apresentação da revisão $0^{5,6}$.

O questionamento direcionador para a presente pesquisa foi: Qual a produção de conhecimentos sobre qualidade da voz e lesões das pregas vocais na população pediátrica?

O levantamento dos artigos científicos foi realizado por meio das bases de dados Medline, LILACS, IBECS (por meio do portal BVS), PubMed e ISI Web of Science . Na busca dos artigos foram utilizados os descritores "Distúrbios da Voz", "Disfonia", "Rouquidão", "Qualidade da Voz", "Acústica da Fala", "Voz", "Fonação", "Pregas Vocais", "Pediatria", "Criança", "Pré-Escolar", em inglês, português, espanhol e suas combinações.

Como critérios de inclusão consideraram-se artigos científicos completos publicados nos anos de 2001 a 2016 nos idiomas inglês, português e espanhol, e que abordavam os temas de qualidade vocal e lesões das pregas vocais na população pediátrica. Os critérios definidos para a exclusão das pesquisas foram artigos que possuíam acesso apenas ao resumo, citações repetidas nas bases de dados e que não se relacionavam diretamente ao objetivo da investigação.

Os artigos encontrados na busca foram analisados por duas pesquisadoras, independentemente, quanto à pertinência aos critérios de inclusão no estudo. Foram excluídos os artigos que não se relacionavam diretamente ao tema. Quando houve discordância entre as pesquisadoras sobre a pertinência do artigo, os mesmos foram analisados por consenso.

Para a análise dos dados, as informações coletadas foram organizadas de maneira concisa em um banco de dados, sendo realizada de forma descritiva, considerando as informações referentes à amostra, objetivos, metodologia e resultados principais, e organizadas por similaridade de conteúdo.

Após a análise dos estudos selecionados foi realizada distribuição em categorias, seguindo as características da qualidade vocal em crianças normofônicas e em crianças disfônicas e sobre as características das lesões de pregas vocais mais prevalentes em crianças disfônicas.

\section{Resultado}

Foram identificados 770 artigos, dos quais somente 36 estavam diretamente relacionados à pergunta de pesquisa e foram analisados. Os resultados da seleção e análise da pesquisa estão descritos na Figura 1.

Dentre os estudos incluídos na pesquisa bibliográfica, nenhum teve metodologia semelhante, o que impossibilitou a realização de meta-análise dos resultados encontrados.

A análise das publicações permitiu a identificação de duas temáticas principais, observadas na Figura 2: crianças normofônicas (qualidade vocal) e crianças disfônicas (qualidade vocal e lesões de pregas vocais).

Os resultados da revisão de literatura, definindo a casuística e resultados observados, estão apresentados no Quadro 1. 


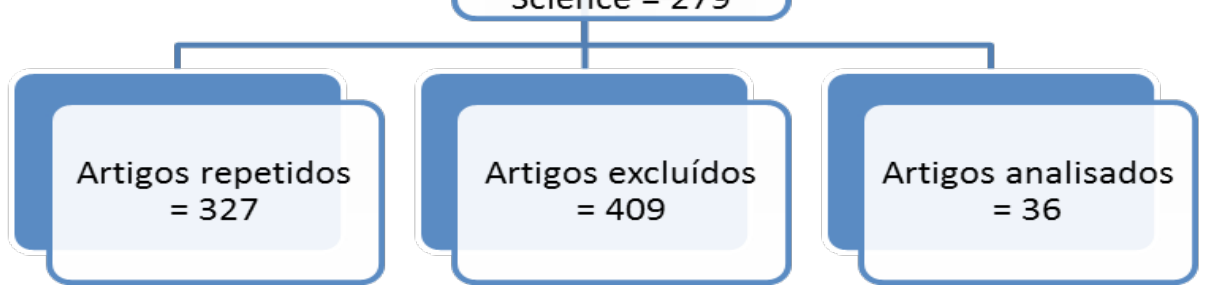

Figura 1. Seleção e análise dos artigos

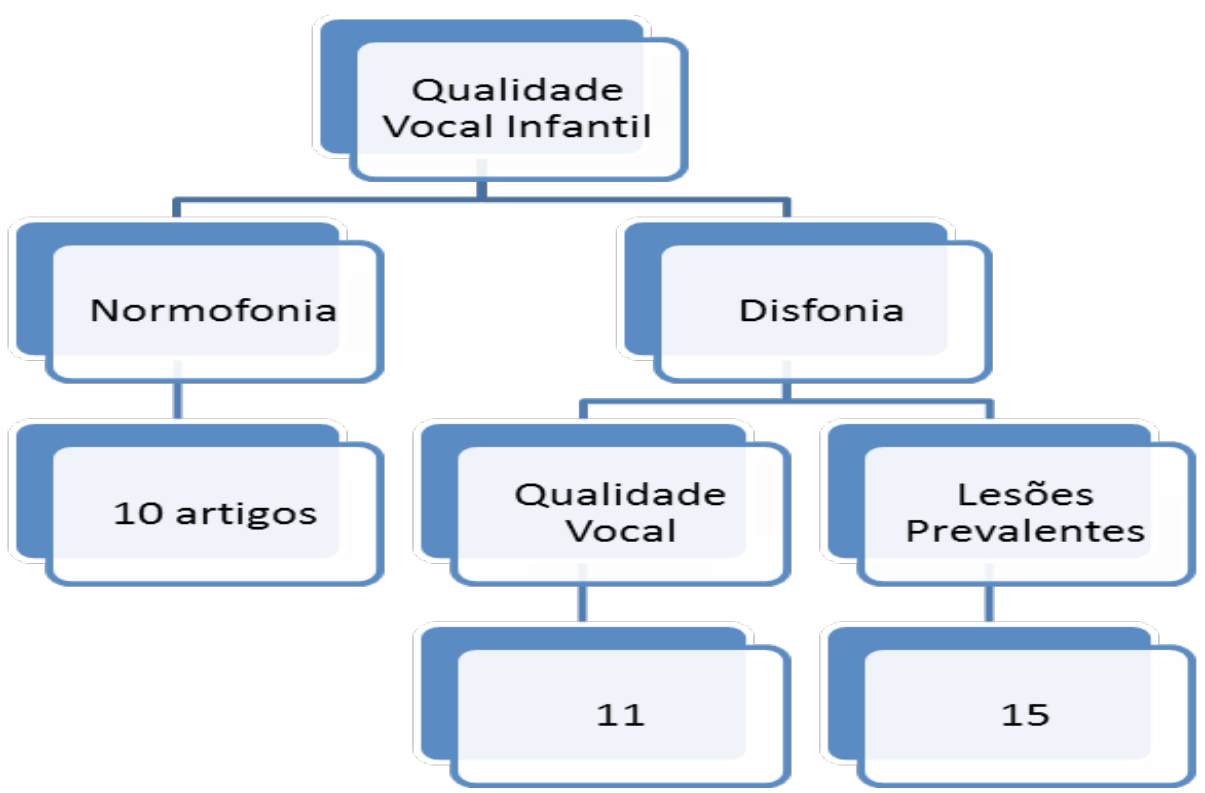

Figura 2. Artigos subdivididos por temática

Número de

identificados $=$ 770

$$
\text { BVS }=214
$$

PubMed $=277$

ISI Web of

Science $=279$ 
Quadro 1. Artigos selecionados para a revisão de literatura.

\begin{tabular}{|c|c|c|c|c|}
\hline AUTORES/ANO & DELINEAMENTO & $\begin{array}{l}\text { NÚMERO DE } \\
\text { SUJEITOS E } \\
\text { IDADE }\end{array}$ & AVALIAÇÃO & RESULTADOS \\
\hline $\begin{array}{c}\text { Oliveira RC, Teixeira } \\
\text { LC, Gama ACC, } \\
\text { Medeiros AM. } \\
(2011)^{1}\end{array}$ & $\begin{array}{c}\text { Transversal com } \\
\text { amostra aleatória }\end{array}$ & $\begin{array}{l}70 \text { crianças com } \\
\text { idade entre seis a } \\
10 \text { anos }\end{array}$ & $\begin{array}{l}\text { Análise perceptivo- } \\
\text { auditiva (escala GRBASI) } \\
\text { Análise acústica }\end{array}$ & $\begin{array}{l}\text { Qualidade vocal mais comum entre as } \\
\text { crianças disfônicas: soprosidade seguida } \\
\text { de rugosidade. Medidas acústicas de } \\
\text { APQ, PPQ e PHR: mais elevadas entre as } \\
\text { crianças disfônicas.. }\end{array}$ \\
\hline $\begin{array}{c}\text { Hill CA, Shilpa O, } \\
\text { Maturo S, Maurer R, } \\
\text { Bunting G, Hartnick } \\
\text { CJ. }(2013)^{7}\end{array}$ & $\begin{array}{l}\text { Prospectivo } \\
\text { longitudinal }\end{array}$ & $\begin{array}{l}50 \text { participantes } \\
\text { com idade entre } \\
\text { quatro a } 17 \text { anos }\end{array}$ & Análise acústica & $\begin{array}{l}\text { Média da frequência fundamental nos } \\
\text { sujeitos de quatro a } 17 \text { anos de idade: } \\
244 \mathrm{~Hz} \text {. }\end{array}$ \\
\hline $\begin{array}{c}\text { Cappellari VM, Cielo } \\
\text { CA. }(2008)^{8}\end{array}$ & $\begin{array}{l}\text { Prospectivo } \\
\text { de coorte } \\
\text { transversal }\end{array}$ & $\begin{array}{l}23 \text { crianças com } \\
\text { idade entre quatro } \\
\text { a oito anos e seis } \\
\text { meses }\end{array}$ & $\begin{array}{l}\text { Avaliação perceptivo- } \\
\text { auditiva (escala RASAT) } \\
\text { Análise acústica }\end{array}$ & $\begin{array}{c}\text { A variação de frequência (vfO) e a } \\
\text { proporção harmônico-ruído (NHR) } \\
\text { foram maiores na amostra total que } \\
\text { aos cinco e seis anos; à medida que } \\
\text { a idade aumentou, o NHR reduziu; à } \\
\text { medida que o PPQ aumentou, a vfo, } \\
\text { variação de amplitude (vAm), o índice } \\
\text { de fonação suave (SPI) e o NHR também } \\
\text { aumentaram; à medida que o PPQ, APQ } \\
\text { e o índice de turbulência vocal (VTI) } \\
\text { aumentaram, o índice de fonação suave } \\
\text { (SPI) reduziu. }\end{array}$ \\
\hline $\begin{array}{c}\text { Viegas F, Viegas D, } \\
\text { Atherino CCT, Baeck } \\
\text { HE. }(2010)^{9}\end{array}$ & $\begin{array}{l}\text { Coorte trans- } \\
\text { versal }\end{array}$ & $\begin{array}{l}207 \text { crianças com } \\
\text { idade entre quatro } \\
\text { a oito anos }\end{array}$ & $\begin{array}{l}\text { Análise perceptivo- } \\
\text { auditiva Análise acústica }\end{array}$ & $\begin{array}{c}\text { Decréscimo na frequência fundamental } \\
\text { com o aumento da idade. A idade de seis } \\
\text { anos foi apontada como determinante } \\
\text { para as mudanças acústicas das } \\
\text { vocalizações infantis. }\end{array}$ \\
\hline $\begin{array}{c}\text { Infusino SA, Diercks } \\
\text { GR, Rogers, } \\
\text { DJ, Garcia J, Ojha } \\
\text { S, Maurer R, Bunting } \\
\text { G, Hartnick CJ. } \\
(2015)^{10}\end{array}$ & Corte transversal & $\begin{array}{l}218 \text { participantes } \\
\text { com idade entre } \\
\text { quatro a } 17 \text { anos }\end{array}$ & Análise acústica & $\begin{array}{c}\text { Redução significativa na frequência } \\
\text { fundamental nos meninos normofônicos } \\
\text { nas idades entre } 11 \text { e } 14 \text { anos. Nas } \\
\text { meninas observou-se tendência } \\
\text { de diminuição linear na frequência } \\
\text { fundamental, sem nenhum marco etário. }\end{array}$ \\
\hline Hunter EJ. (2009) ${ }^{11}$ & Estudo de caso & $\begin{array}{l}\text { Uma criança com } \\
\text { cinco anos e sete } \\
\text { meses de idade }\end{array}$ & Análise acústica & $\begin{array}{l}\text { Os resultados deste estudo mostram } \\
\text { que as crianças podem produzir uma } \\
\text { voz notavelmente diferente durante as } \\
\text { observações clínicas em comparação com } \\
\text { as atividades diárias de rotina. }\end{array}$ \\
\hline $\begin{array}{l}\text { McAllister A, Brandt } \\
\text { SK. }(2012)^{12}\end{array}$ & Experimental & $\begin{array}{l}11 \text { crianças com } \\
\text { cinco anos de } \\
\text { idade }\end{array}$ & Análise acústica & $\begin{array}{c}\text { Quando comparado as médias da } \\
\text { frequência fundamental em uma emissão } \\
\text { controlada e na fala espontânea, esta } \\
\text { foi significantemente maior na fala } \\
\text { espontânea. Os resultados sugerem que } \\
\text { as condições de gravação controladas } \\
\text { podem ser inadequadas para caracterizar } \\
\text { o comportamento vocal das crianças em } \\
\text { um ambiente natural. }\end{array}$ \\
\hline $\begin{array}{l}\text { Baker S, Weinrich } \\
\text { B, Bevington } \\
\text { M, Schroth K, } \\
\text { Schroeder E. } \\
(2008)^{13}\end{array}$ & Experimental & $\begin{array}{l}48 \text { participantes } \\
\text { com idade entre } \\
\text { seis a } 10 \text { anos }\end{array}$ & Análise acústica & $\begin{array}{l}\text { Os resultados revelaram que o tipo de } \\
\text { tarefa influencia significativamente os } \\
\text { valores da frequência fundamental em } \\
\text { criança. A frequência fundamental foi } \\
\text { maior na contagem numérica e na vogal } \\
\text { sustentada quando comparadas à frase e } \\
\text { à sentença. }\end{array}$ \\
\hline $\begin{array}{c}\text { Brockmann- } \\
\text { Bauser M, Beyer } \\
\text { D, Bohlender JE } \\
(2015)^{14}\end{array}$ & $\begin{array}{l}\text { Coorte trans- } \\
\text { versal }\end{array}$ & $\begin{array}{l}68 \text { crianças com } \\
\text { idade entre cinco } \\
\text { a nove anos e } 11 \\
\text { meses }\end{array}$ & Análise acústica & $\begin{array}{l}\text { Em crianças normofônicas as medidas } \\
\text { acústicas de frequência fundamental } \\
\text {,jitter, e shimmer foram diferentes na } \\
\text { emissão habitual em comparação com } \\
\text { a emissão controlada. A frequência } \\
\text { fundamental foi maior e as medidas } \\
\text { de jitter e shimmer foram menores } \\
\text { em crianças do sexo feminino. As } \\
\text { variáveis sexo, idade, altura e peso não } \\
\text { interferiram nos valores de frequência } \\
\text { fundamental , jitter, shimmer nas } \\
\text { emissões controladas. }\end{array}$ \\
\hline $\begin{array}{c}\text { Brockmann- } \\
\text { Bauser M, Beyer } \\
\text { D, Bohlender JE } \\
(2014)^{15}\end{array}$ & Corte transversal & $\begin{array}{l}49 \text { crianças com } \\
\text { idade entre cinco } \\
\text { a nove anos e } 11 \\
\text { meses }\end{array}$ & Análise Acústica & $\begin{array}{c}\text { Ao comparar emissões habituais e } \\
\text { emissões controladas de crianças sem } \\
\text { alterações, observou se que as medidas } \\
\text { acústicas de jitter e shimmer obtiveram } \\
\text { valores menores nas emissões realizadas } \\
\text { na intensidade de } 80 \mathrm{~dB} \text {. }\end{array}$ \\
\hline
\end{tabular}




\begin{tabular}{|c|c|c|c|c|}
\hline AUTORES/ANO & DELINEAMENTO & $\begin{array}{l}\text { NÚMERO DE } \\
\text { SUJEITOS E } \\
\text { IDADE }\end{array}$ & AVALIAÇÃO & RESULTADOS \\
\hline $\begin{array}{l}\text { Tavares EM, Labio } \\
\text { RB, Martins RHG } \\
\quad(2010)^{16}\end{array}$ & Corte transversal & $\begin{array}{l}240 \text { participantes } \\
\text { com idade entre } \\
\text { quatro a } 12 \text { anos }\end{array}$ & $\begin{array}{c}\text { Análise perceptivo- } \\
\text { auditiva Análise Acústica } \\
\text { Videolaringoestroboscopia }\end{array}$ & $\begin{array}{l}\text { Com o aumento da idade, houve redução } \\
\text { das medidas acústicas d frequência } \\
\text { fundamental e APQ e aumento da medida } \\
\text { acústica de SPI. Os parâmetros vocais } \\
\text { não diferiram entre os gêneros até a } \\
\text { idade de } 12 \text { anos. }\end{array}$ \\
\hline $\begin{array}{l}\text { Cielo CA, Cappellari } \\
\text { VM. }(2008)^{17}\end{array}$ & $\begin{array}{l}\text { Prospectivo } \\
\text { de coorte } \\
\text { transversal }\end{array}$ & $\begin{array}{c}23 \text { crianças com } \\
\text { idade entre quatro } \\
\text { a seis anos e oito } \\
\text { meses }\end{array}$ & $\begin{array}{l}\text { Avaliação perceptivo- } \\
\text { auditiva (escala RASAT) }\end{array}$ & $\begin{array}{l}\text { Os TMF aos seis anos foram maiores do } \\
\text { que aos quatro anos. À medida que a } \\
\text { idade aumentou, todos os valores de TMF } \\
\text { também aumentaram, e a relação s/z } \\
\text { para todas as idades foi próxima de um. }\end{array}$ \\
\hline $\begin{array}{c}\text { Tavares EM, } \\
\text { Brasolotto A, } \\
\text { Santana MF, } \\
\text { Padovan CA, Martins } \\
\text { RHG. }(2009)^{18}\end{array}$ & $\begin{array}{l}\text { Analítico } \\
\text { transversal }\end{array}$ & $\begin{array}{l}2.000 \text { crianças } \\
\text { com idade entre } \\
\text { quatro a } 12 \text { anos }\end{array}$ & $\begin{array}{c}\text { Análise perceptivo- } \\
\text { auditiva (escala GRBASI) } \\
\text { Análise acústica Exame } \\
\text { de videolaringoscopia }\end{array}$ & $\begin{array}{c}\text { Houve diminuição da frequência } \\
\text { fundamental com a idade e os demais } \\
\text { parâmetros acústicos mostraram- } \\
\text { se mais elevados nas crianças com } \\
\text { disfonia. Nas laringoscopias observou-se } \\
\text { nódulos, espessamentos e inflamação. O } \\
\text { julgamento dos pais indicou prevalência } \\
\text { de disfonia em } 6,15 \% \text {, e as análises } \\
\text { perceptivas em } 11,4 \% \text {. }\end{array}$ \\
\hline $\begin{array}{l}\text { Simões-Zenari M, } \\
\text { Nemr K, Behlau M } \\
(2012)^{19}\end{array}$ & Corte transversal & $\begin{array}{l}100 \text { participantes } \\
\text { com idade entre } \\
\text { quatro a seis anos } \\
\text { e } 11 \text { meses }\end{array}$ & $\begin{array}{l}\text { Análise perceptivo- } \\
\text { auditiva Análise Acústica }\end{array}$ & $\begin{array}{c}\text { O grupo de crianças disfônicas apresentou } \\
\text { maior prevalência de soprosidade e } \\
\text { rugosidade. A frequência fundamental } \\
\text { foi menor no grupo de disfônicos e a } \\
\text { componente de ruído foi mais elevada } \\
\text { nesse grupo. } \\
\end{array}$ \\
\hline $\begin{array}{l}\text { Lopes LW, Lima } \\
\text { ILB, Almeida } \\
\text { LNA, Cavalcante } \\
\text { DP, Almeida AAF. } \\
\quad(2012)^{20}\end{array}$ & $\begin{array}{l}\text { Coorte trans- } \\
\text { versal }\end{array}$ & $\begin{array}{l}71 \text { crianças com } \\
\text { idade entre três a } \\
10 \text { anos }\end{array}$ & $\begin{array}{l}\text { Análise perceptivo- } \\
\text { auditiva Análise acústica }\end{array}$ & $\begin{array}{c}\text { Nenhuma das crianças apresentou } \\
\text { desvio vocal intenso. Observou-se maior } \\
\text { ocorrência de desvio leve nos parâmetros } \\
\text { instabilidade, rugosidade e tensão. O } \\
\text { grau geral moderado, a rugosidade e a } \\
\text { soprosidade foram correlacionados com } \\
\text { maiores valores de shimmer e menor } \\
\text { valor de GNE. }\end{array}$ \\
\hline $\begin{array}{l}\text { Ingrid V, Dominique } \\
\text { M, Marc R. }(2012)^{21}\end{array}$ & Experimental. & \begin{tabular}{|c|}
42 crianças com \\
idade entre cinco a \\
13 anos
\end{tabular} & $\begin{array}{l}\text { Pediatric Voice Symptom } \\
\text { Questionnaire (PVSQ) }\end{array}$ & $\begin{array}{c}\text { O questionário PVSQ é um instrumento } \\
\text { válido e confiável para a autoavaliação da } \\
\text { disfonia na população infantil. }\end{array}$ \\
\hline $\begin{array}{l}\text { Johnson K, Brehm } \\
\text { SK, Weinrich B, } \\
\text { Meinzen-Derr J, } \\
\text { Alarcon A. }(2011)^{22}\end{array}$ & $\begin{array}{l}\text { Retrospectivo } \\
\text { observacional }\end{array}$ & $\begin{array}{l}38 \text { participantes } \\
\text { com idade entre } \\
\text { quatro anos e dois } \\
\text { meses a } 17 \text { anos e } \\
\quad \text { dois meses }\end{array}$ & $\begin{array}{l}\text { Pediatric Voice Handicap } \\
\text { Index (pVHI) Análise } \\
\text { perceptivo-auditiva } \\
\text { (escala CAPE-V) }\end{array}$ & $\begin{array}{c}\text { Observou-se correlação entre o CAPE-V e } \\
\text { o pVHI funcional e entre CAPE-V, tensão } \\
\text { e soprosidade, e o pVHI total e funcional. } \\
\text { O parâmetro perceptivo-auditivo com } \\
\text { maior grau de alteração é a soprosidade } \\
\text { seguida da rugosidade, embora a tensão } \\
\text { e instabilidade também se encontrem } \\
\text { alteradas. }\end{array}$ \\
\hline $\begin{array}{l}\text { Shah RK, Engel SH, } \\
\text { Choi SS. }(2008)^{23}\end{array}$ & $\begin{array}{l}\text { Retrospectivo } \\
\text { longitudinal }\end{array}$ & $\begin{array}{c}40 \text { crianças com a } \\
\text { média de idade de } \\
7,5 \text { anos }\end{array}$ & $\begin{array}{l}\text { Análise perceptivo- } \\
\text { auditiva Análise acústica }\end{array}$ & $\begin{array}{l}\text { Não houve associação entre o tamanho } \\
\text { de nódulos e a frequência fundamental, } \\
\text { o shimmer, a diminuição do suporte } \\
\text { respiratório, a perda de ar ou à tensão } \\
\text { muscular. O tamanho dos nódulos vocais } \\
\text { influencia no pitch, sendo que quanto } \\
\text { maior a lesão mais grave é o pitch. }\end{array}$ \\
\hline $\begin{array}{l}\text { Nardone HC, Recko } \\
\text { T, Huang L, Nuss RC } \\
(2014)^{24}\end{array}$ & $\begin{array}{l}\text { Clínico } \\
\text { retrospectivo }\end{array}$ & \begin{tabular}{|c|}
67 participantes \\
com idade entre \\
três anos e oito \\
meses a 20 anos e \\
seis meses \\
\end{tabular} & $\begin{array}{c}\text { Análise perceptivo- } \\
\quad \text { auditiva } \\
\text { Videolaringoestroboscopia }\end{array}$ & $\begin{array}{l}\text { Crianças com nódulos de pregas vocais } \\
\text { possuem grau de alteração da qualidade } \\
\text { vocal proporcional ao tamanho lesão. }\end{array}$ \\
\hline $\begin{array}{l}\text { Lopes LW, Lima } \\
\text { IL, Barbosa AE, } \\
\text { Heitmann M, Silva } \\
\text { MF, Bonfim L, Silva } \\
\text { POC }(2015)^{25}\end{array}$ & $\begin{array}{l}\text { Analítico } \\
\text { transversal }\end{array}$ & $\begin{array}{l}93 \text { participantes } \\
\text { com idade entre } \\
\text { oito a } 10 \text { anos }\end{array}$ & $\begin{array}{c}\text { Análise perceptivo- } \\
\text { auditiva (escala } \\
\text { analógico- visual) Análise } \\
\text { acústica }\end{array}$ & $\begin{array}{c}\text { 74,1\% apresentaram alteração vocal de } \\
\text { grau leve nos parâmetros rugosidade } \\
\text { e instabilidade. Em } 14 \% \text { observou-se } \\
\text { desvio de grau moderado e apenas } \\
11,9 \% \text { dos sujeitos apresentaram voz } \\
\text { saudável. }\end{array}$ \\
\hline $\begin{array}{l}\text { Reynolds V, } \\
\text { Buckland A, Bailey J, } \\
\text { Lipscombe J, Nathan } \\
\text { E, Vijayasekaran S, } \\
\text { Kelly R, Maryn Y, } \\
\text { French N. }(2012)^{26}\end{array}$ & $\begin{array}{l}\text { Prospectivo } \\
\text { observacional }\end{array}$ & $\begin{array}{l}97 \text { crianças com } \\
\text { idade entre seis a } \\
15 \text { anos }\end{array}$ & $\begin{array}{l}\text { Análise perceptivo- } \\
\text { auditiva Análise acústica } \\
\text { por meio do The Acoustic } \\
\text { Voice Quality Index } \\
\text { (AVQI) }\end{array}$ & $\begin{array}{l}\text { Os indivíduos pré-termo extremos } \\
\text { possuem maiores alterações na análise } \\
\text { acústica e perceptivo-auditiva quando } \\
\text { comparados às crianças nascidas a } \\
\text { termo. O AVQI tem uma precisão de } \\
\text { diagnóstico na população infantil, o que } \\
\text { sugere que ele é uma ferramenta de } \\
\text { avaliação adequada para determinar a } \\
\text { presença e grau de desvios de distúrbios } \\
\text { da voz pediátricos. }\end{array}$ \\
\hline
\end{tabular}




\begin{tabular}{|c|c|c|c|c|}
\hline AUTORES/ANO & DELINEAMENTO & $\begin{array}{l}\text { NÚMERO DE } \\
\text { SUJEITOS E } \\
\text { IDADE }\end{array}$ & AVALIAÇÃo & RESULTADOS \\
\hline $\begin{array}{l}\text { Walz PC, Hubbell, } \\
\text { MP, Elmaraghy, CA } \\
(2014)^{27}\end{array}$ & $\begin{array}{l}\text { Prospectivo } \\
\text { observacional }\end{array}$ & $\begin{array}{l}69 \text { crianças com a } \\
\text { média de idade de } \\
28 \text { anos }\end{array}$ & $\begin{array}{l}\text { Pediatric Voice Outcomes } \\
\text { Survey (pVOS) Pediatric } \\
\text { Voice-Related Quality of } \\
\text { Life (pVRQOL) }\end{array}$ & $\begin{array}{l}\text { Os pacientes pré-termo com maior tempo } \\
\text { de intubação possuem impacto negativo } \\
\text { na qualidade da voz. A pontuação } \\
\text { pVRQOL foi correlacionada positivamente } \\
\text { com idade gestacional e negativamente } \\
\text { correlacionada com o tempo da } \\
\text { intubação. } \\
\end{array}$ \\
\hline $\begin{array}{l}\text { Lábio RB, Tavares } \\
\text { ELM, Alvarado } \\
\text { RC, Martins RHG. } \\
\quad(2012)^{28}\end{array}$ & $\begin{array}{l}\text { Retrospectivo } \\
\text { oberservacional }\end{array}$ & $\begin{array}{c}120 \text { crianças com } \\
\text { idade entre quatro } \\
\text { a } 12 \text { anos }\end{array}$ & $\begin{array}{c}\text { Análise perceptivo- } \\
\text { auditiva Análise acústica }\end{array}$ & $\begin{array}{l}\text { A qualidade vocal de crianças com } \\
\text { obstrução de via aérea superior tem grau } \\
\text { aumentado de hiponasalidade, pitch mais } \\
\text { grave e voz comprimida. Na avaliação } \\
\text { acústica observa-se que a frequência } \\
\text { fundamental é sempre menor no grupo } \\
\text { com obstrução nasal crônica e as medidas } \\
\text { de jitter, shimmer e PHR são melhores no } \\
\text { grupo sem obstrução nasal. }\end{array}$ \\
\hline $\begin{array}{c}\text { Lundeborg I, } \\
\text { Hultcrantz } E_{,} \\
\text {Ericsson } E, \text { McAllister } \\
\text { A. }(2012)^{29} \\
\end{array}$ & $\begin{array}{l}\text { Retrospectivo } \\
\text { clínico } \\
\text { randomizado }\end{array}$ & $\begin{array}{l}189 \text { crianças com } \\
\text { idade entre } 50 \text { a } \\
65 \text { meses }\end{array}$ & $\begin{array}{l}\text { Análise perceptivo- } \\
\text { auditiva Análise acústica }\end{array}$ & $\begin{array}{l}\text { A qualidade vocal é afetada } \\
\text { perceptivamente e acusticamente pela } \\
\text { hipertrofia adenoamigdaliana. Após a } \\
\text { cirurgia a voz é normalizada, mas as } \\
\text { diferenças acústicas permanecem. }\end{array}$ \\
\hline $\begin{array}{l}\text { Gramuglia ACJ, } \\
\text { Tavares ELM, } \\
\text { Rodrigues AS, } \\
\text { Martins RHG } \\
(2014)^{30}\end{array}$ & Corte transversal & $\begin{array}{l}200 \text { participantes } \\
\text { com idade entre } \\
\text { quatro a } 11 \text { anos }\end{array}$ & $\begin{array}{c}\text { Análise perceptivo- } \\
\text { auditiva Análise Acústica } \\
\text { Videolaringoestroboscopia }\end{array}$ & $\begin{array}{c}\text { Ao comparar as vozes de crianças com } \\
\text { nódulos de pregas vocais com crianças } \\
\text { sem alteraç̃os laríngeas observou-se } \\
\text { que o grau de alteração, a rugosidade e } \\
\text { a soprosidade foram maiores em crianças } \\
\text { com lesão. As medidas acústicas de jitter, } \\
\text { shimmer, PPQ, APQ e NHR foram mais } \\
\text { elevadas no grupo com nódulos vocais. } \\
\text { Não houve diferença dos valores de } \\
\text { frequência fundamental. }\end{array}$ \\
\hline $\begin{array}{l}\text { Garcia-Real T, Diaz- } \\
\text { Roman TM, Garcia- } \\
\text { Martinez V, Vieiro- } \\
\text { Iglesias P }(2013)^{31} \\
\end{array}$ & Corte transversal & $\begin{array}{c}51 \text { crianças com } \\
\text { idade entre seis a } \\
15 \text { anos }\end{array}$ & $\begin{array}{l}\text { Análise perceptivo- } \\
\text { auditiva Análise Acústica }\end{array}$ & $\begin{array}{l}\text { As crianças com diagnóstico de TDAH } \\
\text { apresentaram rouquidão, e tensão } \\
\text { cervical quando comparadas às crianças } \\
\text { do grupo controle. }\end{array}$ \\
\hline $\begin{array}{l}\text { Beber BC, Cielo } \\
\text { CA, Siqueira MA. } \\
\quad(2009)^{32}\end{array}$ & $\begin{array}{l}\text { Prospectivo } \\
\text { oberservacional }\end{array}$ & $\begin{array}{c}54 \text { crianças com } \\
\text { idade entre } 13 \text { a } \\
15 \text { anos }\end{array}$ & $\begin{array}{l}\text { Análise perceptivo- } \\
\text { auditiva }\end{array}$ & $\begin{array}{l}\text { Nas crianças do gênero feminino os } \\
\text { tempos máximos de fonação foram } \\
\text { considerados reduzidos. Nos meninos } \\
\text { com lesões de massa, } 50 \% \text { das crianças } \\
\text { tiveram TMF considerados normais e } 50 \% \\
\text { reduzidos. } \\
\end{array}$ \\
\hline $\begin{array}{l}\text { Melo ECM, Mattioli } \\
\text { FM, Brasil OC, } \\
\text { Behlau M, Pitaluga } \\
\text { ACA, Melo DM. } \\
(2001)^{33}\end{array}$ & $\begin{array}{l}\text { Retrospectivo } \\
\text { clínico não } \\
\text { randomizado }\end{array}$ & $\begin{array}{l}34 \text { crianças com } \\
\text { idade entre quatro } \\
\text { a } 13 \text { anos }\end{array}$ & Videolaringoscopia & $\begin{array}{l}\text { O nódulo vocal foi a lesão mais comum } \\
\text { observada nas crianças avaliadas, sem } \\
\text { prevalência quanto ao sexo, com uma } \\
\text { média de idade de } 9 \text { anos. }\end{array}$ \\
\hline $\begin{array}{c}\text { Martins RHG, Ribeiro } \\
\text { CBH, Mello BMZ, } \\
\text { Branco A, Tavares } \\
\text { ELM. }(2012)^{34} \\
\end{array}$ & $\begin{array}{l}\text { Clínico } \\
\text { prospectivo }\end{array}$ & $\begin{array}{l}304 \text { participantes } \\
\text { com idade entre } \\
\text { quatro a } 18 \text { anos }\end{array}$ & Videolaringoestroboscopia & $\begin{array}{l}\text { A disfonia ocorreu em crianças de } 7 \text { a } 12 \\
\text { anos, no sexo masculino. As lesões mais } \\
\text { observadas foram nódulos vocais e cistos } \\
\text { epidermóides. }\end{array}$ \\
\hline $\begin{array}{c}\text { Connelly A, Clement } \\
\text { WA, Kubba H. } \\
(2009)^{35}\end{array}$ & $\begin{array}{l}\text { Clínico } \\
\text { retrospectivo }\end{array}$ & $\begin{array}{c}142 \text { crianças com } \\
\text { idade entre sete a } \\
12 \text { anos }\end{array}$ & $\begin{array}{l}\text { Espelho laríngeo, } \\
\text { laringoscopia e } \\
\text { microlaringoscopia- } \\
\text { broncoscopia }\end{array}$ & $\begin{array}{l}\text { O cisto epidermóide está relacionado } \\
\text { às disfonias congênitas, presente } \\
\text { em } 7 \% \text { dos casos. O abuso vocal } \\
\text { é um dos fatores que se associam } \\
\text { ao desenvolvimento de lesões por } \\
\text { fonotrauma e, mais especificamente, dos } \\
\text { nódulos vocais. }\end{array}$ \\
\hline $\begin{array}{c}\text { Mortensen M, } \\
\text { Schaberg M, Woo P. } \\
(2010)^{36}\end{array}$ & $\begin{array}{l}\text { Clínico } \\
\text { retrospectivo }\end{array}$ & $\begin{array}{l}80 \text { participantes } \\
\text { com idade entre } \\
\text { três a } 17 \text { anos }\end{array}$ & Videolaringoestroboscopia & $\begin{array}{l}\text { Em crianças com idade inferior a seis } \\
\text { anos a principal causa de alterações } \\
\text { vocais é a presença de alterações } \\
\text { estruturais mínimas. }\end{array}$ \\
\hline $\begin{array}{c}\text { Kallvik E, } \\
\text { LindströmE, } \\
\text { Holmqvist } \mathrm{S}_{\text {, }} \\
\text { Lindman J, Simberg } \\
\mathrm{S}(2015)^{37}\end{array}$ & Corte transversal & $\begin{array}{c}217 \text { crianças com } \\
\text { idade entre seis a } \\
10 \text { anos }\end{array}$ & $\begin{array}{l}\text { Análise perceptivo- } \\
\text { auditiva (escala } \\
\text { analógico- visual). }\end{array}$ & $\begin{array}{l}\text { A prevalência de disfonia para crianças } \\
\text { em idade escolar foi de } 12,0 \% \text {. Nas } \\
\text { meninas, a prevalência foi de } 7,8 \% \text { e } \\
\text { para os menino } 15,8 \% \text {. A rugosidade } \\
\text { foi o parâmetro perceptivo-auditvo mais } \\
\text { prevalente. }\end{array}$ \\
\hline $\begin{array}{c}\text { Stivanin L, Santos } \\
\text { FP, Oliveira CCC, } \\
\text { Santos B, Ribeiro ST, } \\
\text { Scivoletto S }(2015)^{38}\end{array}$ & Corte transversal & $\begin{array}{l}136 \text { crianças com } \\
\text { idade média de } 10 \\
\text { anos e dois meses }\end{array}$ & $\begin{array}{c}\text { Análise perceptivo- } \\
\text { auditiva (escala GRBASI) }\end{array}$ & $\begin{array}{c}\text { Alterações vocais estiveram presentes em } \\
67,6 \% \text {, dos pacientes vítimas de maus } \\
\text { tratos atendidos em um centro de saúde. } \\
\text { A prevalência de alterações vocais foo } \\
\text { maior do que a observada na população } \\
\text { geral. }\end{array}$ \\
\hline
\end{tabular}




\begin{tabular}{|c|c|c|c|c|}
\hline AUTORES/ANO & DELINEAMENTO & $\begin{array}{l}\text { NÚMERO DE } \\
\text { SUJEITOS E } \\
\text { IDADE }\end{array}$ & AVALIAÇÃo & RESULTADOS \\
\hline $\begin{array}{c}\text { Smillie IMK, Cohen } \\
\text { W, Lawson E, Wynne } \\
\text { DM }(2014)^{39}\end{array}$ & $\begin{array}{c}\text { Clínico } \\
\text { prospectivo }\end{array}$ & $\begin{array}{l}195 \text { participantes } \\
\text { com cinco, oito e } \\
11 \text { anos de idade }\end{array}$ & $\begin{array}{c}\text { Análise perceptivo- } \\
\text { auditiva (escala GRBASI) } \\
\text { Protocolo de Qualidade } \\
\text { de Vida e voz Pediátrico } \\
\text { (PVRQOL) }\end{array}$ & $\begin{array}{l}52 \% \text { das crianças obtiveram diagnóstico } \\
\text { de nódulos de pregas vocais com } \\
\text { predomínio no sexo masculino. A } \\
\text { Síndrome da tensão músculo esquelética } \\
\text { esteve presente em } 69 \% \text { dos casos de } \\
\text { disfonia. }\end{array}$ \\
\hline $\begin{array}{c}\text { Mackiewicz- } \\
\text { Nartowicz H., } \\
\text { Sinkiewicz A, } \\
\text { Bielecka A. }(2011)^{40} \\
\end{array}$ & Corte transversal & $\begin{array}{c}150 \text { crianças } \\
\text { com idade entre } \\
\text { dois anos e cinco } \\
\text { meses a } 14 \text { anos } \\
\end{array}$ & Videolaringoestroboscopia & $\begin{array}{c}56,7 \% \text { das crianças apresentaram } \\
\text { nódulos de pregas vocais. A disfonia } \\
\text { funcional foi diagnosticada no restante } \\
\text { (40\% das crianças). }\end{array}$ \\
\hline $\begin{array}{c}\text { Bhattacharyya N } \\
(2015)^{42}\end{array}$ & $\begin{array}{c}\text { Clínico } \\
\text { prospectivo }\end{array}$ & $\begin{array}{l}839 \pm 89 \text { mil } \\
\text { participantes com } \\
\text { idade entre três a } \\
17 \text { anos }\end{array}$ & Questionário específico & $\begin{array}{c}\text { Cerca de } 839 \text { mil crianças }(1,4 \%) \\
\text { atendidas em um centro de saúde no ano } \\
\text { de } 2012 \text { relataram problema de voz. No } \\
\text { geral, } 53,5 \% \text { receberam um diagnóstico. } \\
\text { A laringite }(16,6 \%) \text { e alergias }(10,4 \%) \\
\text { foram os diagnósticos mais comuns. Um } \\
\text { total de } 16,4 \% \text { classificaram o problema } \\
\text { de voz como um "grande" ou "muito } \\
\text { grande". }\end{array}$ \\
\hline $\begin{array}{l}\text { Maia AA, Gama } \\
\text { ACC, Kümmer AM } \\
(2014)^{43}\end{array}$ & $\begin{array}{l}\text { Revisão } \\
\text { integrativa da } \\
\text { literatura }\end{array}$ & $\begin{array}{l}07 \text { artigos com } \\
\text { crianças de idade } \\
\text { entre oito a } 11 \\
\text { anos }\end{array}$ & $\begin{array}{c}\text { Busca de artigos } \\
\text { científicos nas bases } \\
\text { bibliográficas LILACS, } \\
\text { IBECS, MEDLINE, } \\
\text { Biblioteca Cochrane, } \\
\text { SciELO e Web of Science } \\
\text { ISI }\end{array}$ & $\begin{array}{c}\text { A revisão integrativa não estabeleceu } \\
\text { um perfil comportamental em crianças } \\
\text { disfônicas, e não se observou correlação } \\
\text { entre disfonia infantil e comportamento } \\
\text { vocal inadequado. }\end{array}$ \\
\hline
\end{tabular}

\section{Discussão}

\section{Crianças normofônicas}

1. Qualidade vocal

A qualidade vocal de crianças sem alterações vocais e laríngeas esteve presente em dez (29,41\%) estudos incluídos nos critérios dessa revisão.

Uma pesquisa analisou a frequência fundamental (f0) em sujeitos de quatro a 17 anos e obteve a média global de $244 \mathrm{~Hz}^{7}$.

Alguns artigos ${ }^{8,9}$ subdividiram as crianças por grupos etários e as médias da f0 de cada um deles estão descritas na Tabela 1 . Houve consenso entre os resultados, evidenciando que à medida que a idade aumenta, a fo de crianças sem alterações vocais reduz ${ }^{8,9}$. Uma pesquisa observou, ainda, que ao comparar as médias das fo entre os grupos, seis anos é o marco etário para a redução significativa da $\mathrm{f}^{9}$. Outro estudo evidenciou redução na $\mathrm{f0} \mathrm{em}$ meninos com idades entre 11 e $14 \operatorname{anos}^{10}$, caracterizado como o período da muda vocal. A tendência para a diminuição linear na f0 foi encontrada em meninas, contudo, não ocorreu redução significativa em nenhum marco etário ${ }^{10}$. Tais resultados sugerem que o processo de muda vocal ocorre de forma menos acentuada na fo das meninas.

Tabela 1. Frequência fundamental por grupos etários.

\begin{tabular}{cc}
\hline Grupo etário & Média da frequência fundamental (Fo)(08-10) \\
\hline 4 anos a 5 anos e 11 meses & $255 \mathrm{~Hz}$ a $275 \mathrm{~Hz}$ \\
6 anos a 7 anos e 11 meses & $233 \mathrm{~Hz}$ a $248 \mathrm{~Hz}$ \\
8 anos a 9 anos e 11 meses & $230 \mathrm{~Hz}$ a $238 \mathrm{~Hz}$ \\
10 anos a 12 anos & $234 \mathrm{~Hz}$ \\
\hline
\end{tabular}

Dois estudos ${ }^{11,12}$ compararam as médias de f0 na emissão controlada e na fala espontânea e os resultados indicaram que a f0 foi significativamente maior na fala espontânea. Outro estudo ${ }^{13}$ verificou a influência da tarefa solicitada na f0 da voz de crian- ças com idades entre cinco e sete anos e 11 meses sem alteração vocal. A f0 foi consideravelmente maior na contagem numérica e na vogal sustentada, quando comparadas a frases e sentenças ${ }^{13}$. Quando comparadas com a emissão controlada, as 
medidas acústicas de jitter e shimmer em crianças vocalmente saudáveis foram significativamente maiores na emissão habitual ${ }^{14,15}$. Os resultados da literatura evidenciam que as modificações anatômicas sofridas na laringe durante o crescimento infantil modificam a f0, principalmente a partir dos seis anos de idade ${ }^{16}$. Observa-se, também, que a f0 é um parâmetro acústico dependente da tarefa de fala e que a sua análise, considerando a fala encadeada, apresenta-se mais aguda por sofrer influência dos aspectos prosódicos ${ }^{11}$.

As medidas acústicas de aperiodicidade de frequência e intensidade a curto prazo em crianças normofônicas foram também analisadas em três estudos $^{8,9,16}$, por meio do programa Multi Dimensional Voice Program (MDVP) da Kay Pentax ${ }^{\circledR}$, e apresentadas por faixa etária na Tabela 2 .

Tabela 2. Medidas acústicas por grupos etários.

\begin{tabular}{ccccc}
\hline $\begin{array}{c}\text { Medidas } \\
\text { Acústicas }^{(8,9,16)}\end{array}$ & $\mathbf{4}$ anos a $\mathbf{5}$ anos & $\mathbf{6}$ anos a $\mathbf{7}$ anos & $\mathbf{8}$ anos a $\mathbf{9}$ anos & $\mathbf{1 0}$ anos a $\mathbf{1 2}$ anos \\
\hline PPQ\% & 0,85 a $1,21 \%$ & $0,9 \%$ & $0,95 \%$ & $1,01 \%$ \\
APQ\% & 3,13 a $8,1 \%$ & 3,56 a $5,51 \%$ & $3,28 \%$ & $2,85 \%$ \\
PHR & 0,13 a 0,26 & 0,13 a 0,17 & 0,13 & 0,13 \\
\hline
\end{tabular}

Legenda:

PPQ - quociente de perturbação de frequência

$A P Q$ - quociente de perturbação de amplitude

PHR - proporção harmônico ruído

Os resultados da literatura sugerem que as medidas acústicas de quociente de perturbação da frequência (PPQ), quociente de perturbação da amplitude (APQ) e as medidas de ruído, como a Proporção Harmônico Ruído (PHR), não sofrem interferência do desenvolvimento da laringe durante a infância, indicando que a vibração das pregas vocais não se torna mais estável e periódica com a idade . $^{8}$

Os tempos máximos de fonação (TMF) na população pediátrica foram objeto de estudo de duas pesquisas ${ }^{17,18}$, sendo que a primeira analisou os TMF em crianças de quatro a seis anos (Figura 3).

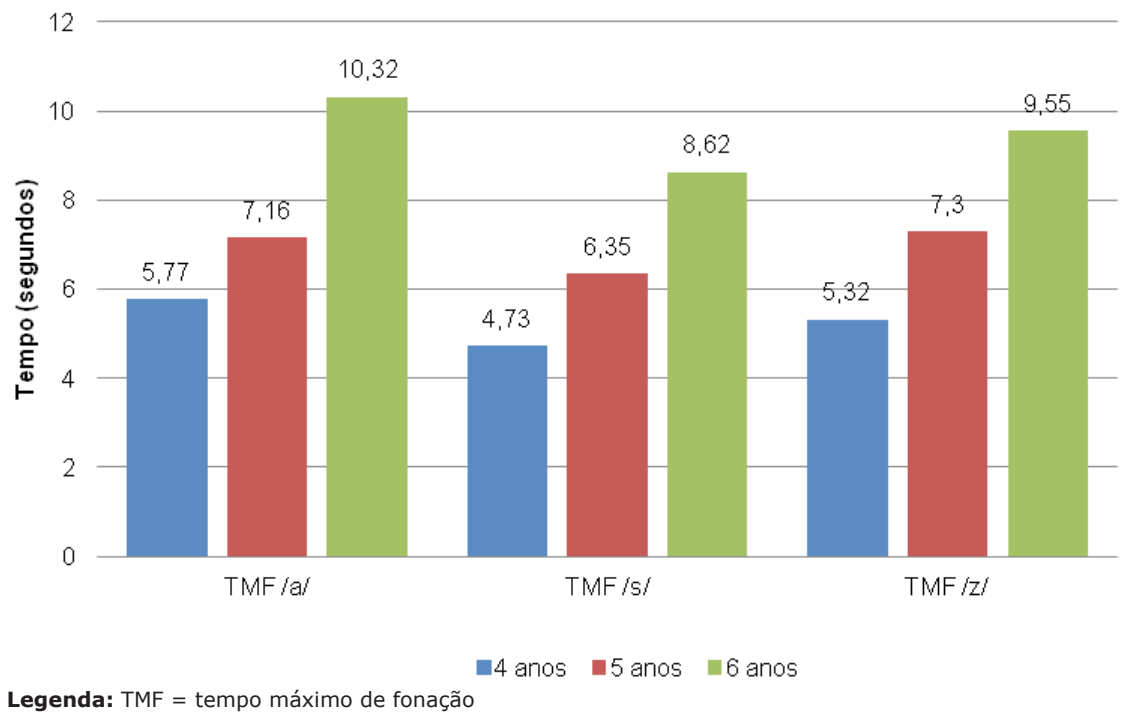

Figura 3. Tempos máximos de fonação por idade ${ }^{(17)}$ 
Ao alocar as crianças em grupos etários de quatro a seis anos, sete a nove anos e 10 a 12 anos, outra pesquisa ${ }^{18}$ observou uma pequena diferença nas médias dos TMF, descritos na Figura 4. Ambos os estudos concluíram que os TMF aumentam proporcionalmente de acordo com a idade, contudo, o aumento significativo foi observado somente até os nove $\operatorname{anos}^{17,18}$. Tais resultados sugerem que o de- senvolvimento anatômico da laringe e dos pulmões impactam positivamente nas medidas dos TMF com aumento progressivo até os nove anos, sendo que a partir desta idade o crescimento anatômico se torna mais lento, com menor interferência nos valores de TMF, que sofrerão maior impacto no período da muda vocal.

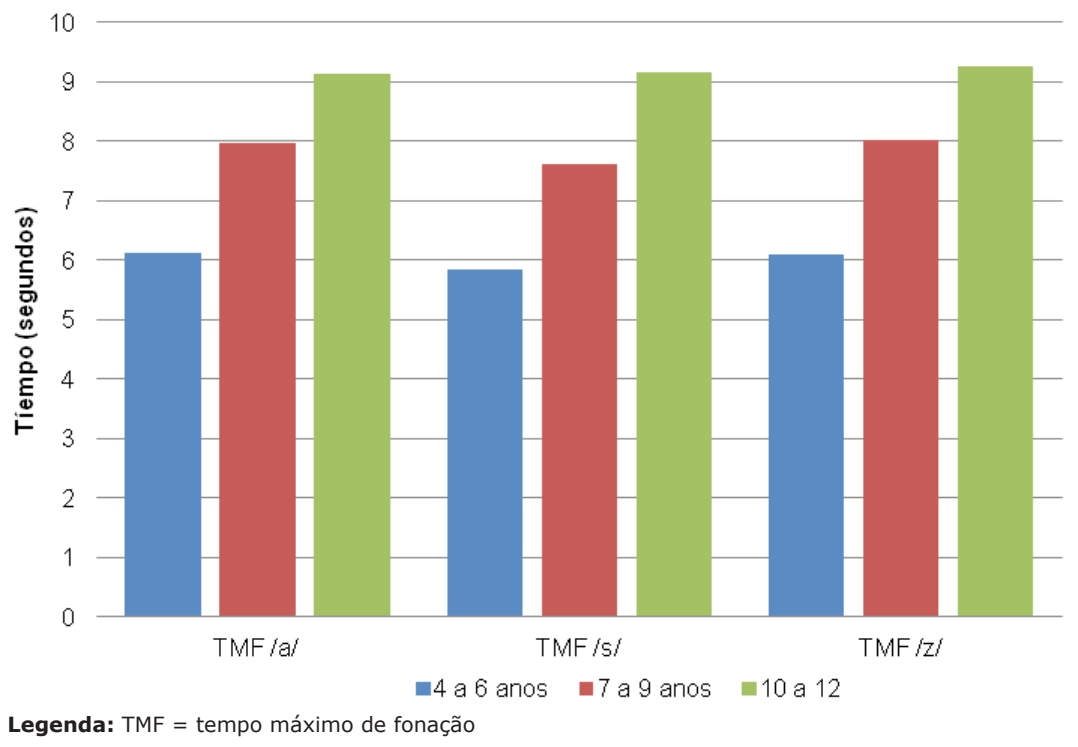

Figura 4. Tempos máximos de fonação por grupos etários(18)

\section{Crianças disfônicas}

\section{Qualidade vocal}

A literatura ${ }^{1,19,20}$ mostra que o parâmetro perceptivo-auditivo com maior grau de alteração em crianças com disfonia organofuncional é a soprosidade, seguido da rugosidade, embora a tensão e a instabilidade também se encontrem alteradas,

A autopercepção vocal negativa é maior em crianças disfônicas do que em crianças sem presença de desvio vocal. O grau geral da disfonia, a rugosidade, soprosidade e tensão afetam, principalmente, o domínio funcional, enquanto a soprosidade influencia, também, o domínio emocional da qualidade de vida ${ }^{1,21,22,23}$.

Dois estudos concluíram que crianças com nódulos de pregas vocais possuem o grau de alteração da qualidade vocal proporcional ao tamanho da lesão nodular ${ }^{24}$.
Um $\operatorname{artigo~}^{25}$ correlacionou o grau das disfonias aos dados perceptivo-auditivos e às medidas acústicas de crianças de três a nove anos de idade. Os resultados demonstraram que $84,5 \%$ das vozes foram classificadas com desvio leve, 2,8\% com desvio moderado e nenhuma com desvio vocal intenso. Quanto aos parâmetros perceptivo-auditivos, a maioria das crianças apresentaram desvio vocal leve nos parâmetros instabilidade (47,88\%), rugosidade $(45,07 \%)$ e tensão $(40,84 \%)$. A rugosidade e a soprosidade foram correlacionados com maiores valores de shimmer e menor valor de $\mathrm{GNE}^{25}$. Nesse estudo foi observado também que crianças com tensão laríngea à fonação obtiveram valores mais elevados de $\mathrm{f0}^{25}$. A justificativa fisiológica para tal achado é que nesses casos há um aumento da tensão longitudinal das pregas vocais e da pressão subglótica que tornam a f0 mais elevada ${ }^{25}$. 
$\mathrm{Na}$ análise acústica do sinal sonoro as medidas APQ, PPQ e PHR apresentaram valores mais elevados em crianças disfônicas ${ }^{1,19}$. Os valores médios da f0 são de $230 \mathrm{~Hz}$ para crianças disfônicas e de $245 \mathrm{~Hz}$ para crianças normofônicas. Observa-se um valor de f0 significativamente menor no grupo de crianças com alterações vocais ${ }^{1,19}$.

A literatura mostra que o tamanho dos nódulos vocais em crianças influencia na percepção do pitch, sendo que quanto maior a lesão mais grave é o pitch ${ }^{23}$.

Dois estudos ${ }^{26,27}$ correlacionaram o grau de alteração da disfonia com prematuridade em crianças disfônicas. Os indivíduos pré-termo extremos possuem maiores alterações na análise acústica e perceptivo-auditiva quando comparados às crianças nascidas a termo ${ }^{26}$. As crianças pré-termo com maior tempo de intubação possuem escores que indicam impacto negativo na qualidade da $\mathrm{voz}^{27}$.

Três artigos compararam a qualidade vocal com a obstrução nasal crônica de crianças saudáveis $^{1,28,29}$. A qualidade vocal de crianças com obstrução de via aérea superior tem grau aumentado de hiponasalidade, pitch mais grave e voz comprimida. $\mathrm{Na}$ avaliação acústica, observa-se que a f0 é menor no grupo com obstrução nasal crônica e as medidas jitter, shimmer e PHR se apresentaram melhores no grupo sem obstrução nasal ${ }^{1,28,29}$.

A literatura ${ }^{1,20,30,31}$ conclui que a voz disfônica na infância é caracterizada por uma qualidade vocal soprosa e rugosa, com grau de alteração leve a moderado. Tais características perceptivo-auditivas são refletidas em medidas acústicas da voz com maior aperiodicidade de frequência (jitter e PPQ), aperiodicidade de intensidade (shimmer e APQ) e maiores medidas de ruído (PHR). A f0 nesta população é mais grave e correlaciona-se com um pitch também grave. Tais resultados sugerem que ambas as formas de avaliação da qualidade vocal, perceptivo-auditiva e acústica, são adequadas na clínica vocal pediátrica, pois representam a voz da criança disfônica.

Somente um artigo analisou a ocorrência dos tipos de TMF (reduzido, normal ou aumentado) em crianças com nódulos e cisto por gênero ${ }^{26}$. Nas crianças do sexo feminino, os TMF foram considerados reduzidos. Nos meninos com lesões de massa, $50 \%$ deles tiveram TMF considerados normais e $50 \%$ reduzidos para a vogal sustentada ${ }^{32}$.

Pode-se concluir que a disfonia na infância promove uma diminuição nos valores de TMF, principalmente nas crianças do sexo feminino. Mais pesquisas sobre as medidas de TMF em crianças disfônicas são importantes para compreender a interferência do gênero nas medidas de TMF.

\section{Lesões de pregas vocais}

A prevalência de lesões de pregas vocais na população pediátrica com disfonia foi objeto de estudo de quinze publicações $(44,1 \%)$. Tais estudos concluíram que o nódulo é a principal causa de alterações vocais infantis, contudo, a prevalência dessas lesões em crianças com queixas vocais variou de $12 \%$ a $94 \% \%^{21,33,41}$.

Alterações vocais estiveram presentes em $67,6 \%$ dos pacientes, vítimas de maus tratos, atendidos em um centro de saúde ${ }^{38}$. A prevalência de alterações vocais encontrada foi maior do que a observada na população geral ${ }^{38}$.

Pesquisas concluíram que em aproximadamente $50 \%$ das crianças a presença de nódulos vocais está associada a abusos vocais ${ }^{36,39,40,41}$, além de causas como sintomas nasais, refluxo faringo-laríngeo e alterações auditivas. Seguidos do nódulo, o cisto epidermoide e o pólipo foram as lesões mais prevalentes, com valores entre $9,9 \%$ a $19 \%{ }^{23-37}$, respectivamente. A presença do cisto epidermoide está altamente relacionada às disfonias congênitas, presente em 7\% dos $\operatorname{casos}^{35}$. Em uma pesquisa que analisou aproximadamente 839 mil crianças atendidas em um centro especializado de saúde no ano de 2012, 1,4\% relataram algum problema de voz. No geral, 53,5\% receberam diagnóstico, sendo a laringite $(16,6 \%)$ e alergias $(10,4 \%)$ os mais comuns ${ }^{4}$.

Dois estudos ${ }^{35,37}$ estratificaram a amostra por grupos etários e os resultados demonstraram que em crianças com idade inferior a seis anos a principal causa de alterações vocais é a presença de alterações estruturais mínimas (AEM). A AEM mais prevalente em crianças é o cisto vocal do tipo epidermoide ${ }^{32-37}$. Uma revisão integrativa de literatura não estabeleceu um perfil comportamental em crianças disfônicas e não encontrou correlação entre a disfonia infantil e o comportamento vocal inadequado ${ }^{43}$. Somente no grupo acima de seis anos houve associação da disfonia com os abusos vocais $^{36}$, que é um dos fatores desencadeantes de lesões por fonotrauma e, mais especificamente, dos nódulos vocais ${ }^{36-41}$.

A literatura ${ }^{35}$ sugere que a lesão de prega vocal mais prevalente nas crianças até seis anos de idade 
é o cisto, sendo que após esta idade o nódulo se torna a lesão mais comum, justificado por fatores comportamentais. A alta variabilidade dos resultados de prevalência encontrados na literatura ${ }^{18,26,27}$ ( $24 \%$ a $94 \%$ ) pode ser explicada pela dificuldade de realização do exame de videolaringoestroboscopia em crianças ${ }^{18}$, já que muitas vezes essas são intolerantes à realização do procedimento e os profissionais nem sempre são especialistas em laringologia pediátrica. Aliado a isso, temos também o fato de que a maioria das pesquisas utilizou amostras de conveniência, com pouca validade externa.

Vale ressaltar que a produção de estudos que investigam a disfonia na infância cresceu na última década.

\section{Conclusão}

Os achados desta revisão demonstram que a qualidade vocal das crianças sem alterações vocais é caracterizada por valores de frequência fundamental que decrescem à medida que a idade aumenta, com diferenças em relação ao sexo e à tarefa de fala. O tempo máximo de fonação também aumenta de acordo com a idade. Estudos mostram que a qualidade vocal característica de crianças disfônicas é soprosa e rugosa com grau de alteração leve a moderado. Em crianças disfônicas, as medidas acústicas da voz e os valores de TMF estão alterados e a f0 é mais grave. Os resultados mostram, também, que a disfonia causa impacto negativo na qualidade de vida da população infantil.

A lesão de prega vocal mais prevalente nas crianças até seis anos de idade é o cisto, sendo que após esta idade o nódulo se torna a lesão mais comumente encontrada, justificado por fatores comportamentais.

\section{Referências bibliográficas}

1. Oliveira RC, Teixeira LC, Gama ACC, Medeiros AM. Análise perceptivo-auditiva, acústica e autopercepção vocal em crianças. J Soc Bras Fonoaudiol. 2011; 23(2): 158-63.

2. Angelillo N, Di Costanzo B, Angelillo M, Costa G, Barillari MR, Barillari U. Epidemiological Study on vocal disorders in pediatric age. J Prev Med Hyg. 2008; 49: 1-5.

3. Behlau M, Madazzio G, Feijó D, Azevedo R, Gielow I, Rehder MI - Aperfeiçoamento Vocal e Tratamento Fonoaudiológico das Disfonias. In: Voz: O livro do especialista. Rio de Janeiro: Revinter; 2005. v.2. p. 409 - 528.
4. Sapienza CM, Ruddy BH, Baker S. Laryngeal Structure and Function in the Pediatric Larynx: Clinical Applications. Lang Speech Hear Serv Sch. 2004; 35: 299-307.

5. Mendes KDS, Silveira RCCP, Galvão CM. Revisão integrativa: método de pesquisa para a incorporação de evidências na saúde e na enfermagem. Texto \& Contexto Enfermagem. 2008; 17(4): 758-64.

6. Souza MT, Silva MD, Carvalho R. Revisão integrativa: o que é e como fazer. Einstein J Biol Med. 2010; 8(1): 102-6.

7. Hill CA, Shilpa O, Maturo S, Maurer R, Bunting G, Hartnick CJ. Consistency of Voice Frequency and Perturbation Measures in Children. JAMA Otolaryngol Head Neck Surg. 2013; 148(4): 637-41.

8. Cappellari VM, Cielo CA. Características vocais acústicas de crianças pré-escolares. Rev Bras Otorrinolaringol. 2008; 74(2): 265-72.

9. Viegas F, Viegas D, Atherino CCT, Baeck HE. Frequência fundamental das 7 vogais orais do português em vozes de crianças. Revista CEFAC, 2010; 12(4): 563-70.

10. Infusino SA, Diercks GR, Rogers, DJ, Garcia J, Ojha S, Maurer R, Bunting G, Hartnick CJ. Establishment of a normative cepstral pediatric acoustic database. JAMA Otolaryngol Head Neck Surg, 2015; 141(4): 358-63.

11. Hunter EJ. A comparison of a child's fundamental frequencies in structured elicited vocalizations versus unstructured natural vocalizations: A case study. Int J Pediatr Otorhinolaryngol. 2009; 73(4): 561-71.

12. McAllister A, Brandt SK. A Comparison of Recordings of Sentences and Spontaneous Speech: Perceptual and Acoustic Measures in Preschool Children's Voices. J Voice. 2012; 26(5): 673.

13. Baker S, Weinrich B, Bevington M, Schroth K, Schroeder E. The effect of task type on fundamental frequency in children. Int J Pediatr Otorhinolaryngol. 2008; 72(1): 885-9.

14. Brockmann-Bauser M, Beyer D, Bohlender JE. Reliable acoustic measurements in children between 5;0 and 9;11 years: Gender, age, height and weight effects on fundamental frequency, jitter and shimmer in phonations without and with controlled voice SPL. Int J Pediatr Otorhinolaryngol. 2015; 79(12): 2035-42.

15. Brockmann-Bauser M, Beyer D, Bohlender JE. Clinical relevance of speaking voice intensity effects on acoustic jitter and shimmer in children between $5 ; 0$ and $9 ; 11$ years. Int J Pediatr Otorhinolaryngol. 2014; 78(12): 2121-6.

16. Tavares EM, Labio RB, Martins RHG. Normative study of vocal acoustic parameters from children from 4 to 12 years of age without vocal symptoms. A pilot study. Braz J Otorhinolaryngol. 2010; 76(4): 485-90.

17. Cielo CA, Cappellari VM. Tempo máximo de fonação de crianças pré-escolares. Rev Bras Otorrinolaringol. 2008; 74(4): 552-60.

18. Tavares EM, Brasolotto A, Santana MF, Padovan CA, Martins RHG. Epidemiological study of dysphonia in 4-12 year-old children. Braz J Otorhinolaryngol. 2011; 77(6): 736-46.

19. Simões-Zenari M, Nem K, Behlau M. Voice disorders in children and its relationship with auditory, acoustic and vocal behavior parameters. Int J Pediatr Otorhinolaryngol. 2012; 76(6): 896-900 
20. Lopes LW, Lima ILB, Almeida LNA, Cavalcante DP Almeida AAF. Análise acústica de vozes infantis: contribuições do diagrama de desvio fonatório. Rev. CEFAC. 2015; 17(4): 1173-83.

21. Ingrid V, Dominique M, Marc R. Symptom Questionnaire: A Double-Form Questionnaire for Dysphonic Children and Their Parents. J Voice. 2012; 26(4): 129-39.

22. Johnson K, Brehm SK, Weinrich B, Meinzen-Derr J, Alarcon A. Comparison of the Pediatric Voice Handicap Index With Perceptual Voice Analysis in Pediatric Patients With Vocal Fold Lesions. Arch Otolaryngol Head Neck Surg. 2011; 137(12): 1258-62.

23. Shah RK, Engel SH, Choi SS. Relationship between voice quality and vocal nodule size. Arch Otolaryngol Head Neck Surg. 2008; 139(5): 723-6.

24. Nardone HC, Recko T, Huang L, Nuss RC. A retrospective review of the progression of pediatric vocal fold nodules. JAMA Otolaryngol Head Neck Surg. 2014; 140(3): 233-6.

25. Lopes LW, Lima ILB, Almeida LNA, Cavalcante DP, Almeida AAF. Severity of Voice Disorders in Children: Correlations between Perceptual and Acoustic Data. J Voice. 2012;26(6):819.

26. Reynolds V, Buckland A, Bailey J, Lipscombe J, Nathan E, Vijayasekaran S, Kelly R, Maryn Y, French N. Objective Assessment of Pediatric Voice Disorders With the Acoustic Voice Quality Index. J Voice. 2012; 26(5): 672.

27. Walz PC, Hubbell, MP, Elmaraghy, CA. Voice related quality of life in pediatric patients with a history of prematurity. Int J Pediatr Otorhinolaryngol. 2014; 78(7): 1010-4.

28. Lábio RB, Tavares ELM, Alvarado RC, Martins RHG. Consequences of Chronic Nasal Obstruction on the Laryngeal Mucosa and Voice Quality of 4- to 12-Year-Old Children. J Voice. 2012;26(4):488-92.

29. Lundeborg I, Hultcrantz E, Ericsson E, McAllister A. Acoustic and Perceptual Aspects of Vocal Function in Children With Adenotonsillar Hypertrophy_Effects of Surgery. J Voice. 2012; 26(4): 480-7.

30. Gramuglia ACJ, Tavares ELM, Rodrigues AS, Martins RHG. Perceptual and acoustic parameters of vocal nodules in children. Int J Pediatr Otorhinolaryngol. 2014; 78(2): 312-6.

31. Garcia-Real T, Diaz-Roman TM, Garcia-Martinez V, VieiroIglesias P. Clinical and acoustic vocal profile in children with attention deficit hyperactivity disorder. J Voice. 2013; 27(6): 787.

32. Beber BC, Cielo CA, Siqueira MA. Lesões de borda de pregas vocais e tempos máximos de fonação. Revista CEFAC. 2009; 11(1): 134-41.

33. Melo ECM, Mattioli FM, Brasil OC, Behlau M, Pitaluga ACA, Melo DM. Disfonia infantil: aspectos epidemiológicos. Rev Bras Otorrinolaringol. 2001; 67(6) :804-7.

34. Martins RHG, Ribeiro CBH, Mello BMZ, Branco A, Tavares ELM. Dysphonia in Children. J Voice. 2012,26(5): 674.

35. Connelly A, Clement WA, Kubba H. Management of dysphonia in children. J Laryngol Otol. 2009; 123(6): 642-7.

36. Mortensen M, Schaberg M, Woo P. Diagnostic Contributions of Videolaryngostroboscopy in the Pediatric Population. Arch Otolaryngol Head Neck Surg. 2010; 136(1): 75-9.
37. Kallvik E, LindströmE, Holmqvist S, Lindman J, Simberg $\mathrm{S}$. Prevalence of hoarseness in school-aged children. J Voice. $2015 ; 29(2): 260$.

38. Stivanin L, Santos FP, Oliveira CCC, Santos B, Ribeiro ST, Scivoletto S. Auditory-perceptual analysis of voice in abused children and adolescents. Braz J OtorhinolaryngoL. 2015; 81(1): 71-8.

39. Smillie IMK, Cohen W, Lawson E, Wynne DM. The paediatric voice clinic. Arch Dis Child. 2014; 99(10): 912-5.

40. Mackiewicz-Nartowicz H, Sinkiewicz A, Bielecka A. Laryngovideostroboscopy in children:Diagnostic possibilities and constraints. Int J Pediatr Otorhinolaryngol. 2011; 75(8): 1015-7.

41. Martins, R.H.G; Ribeiro, C.B.H.; Mello, B.M.Z.; Branco, A.; Tavares,E.L.M. Dysphonia in Children. J Voice. 2012, 26(5):674

42. Bhattacharyya $N$. The prevalence of pediatric voice and swallowing problems in the United States. Laryngoscope. 2015; 125(3): 746-50.

43. Maia AA, Gama ACC, Kümmer AM. Behavioral characteristics of dysphonic children: integrative literature review. CoDAS. 2014; 26(2): 159-63. 